\section{Probing Ribosomal RNA By Electron Spectroscopic Imaging and Three-Dimensional Reconstruction.}

Daniel R. Beniac', Gregory J. Czarnota ${ }^{2}$, Brenda L.

Rutherford $^{2}$, F. Peter Ottensmeyer ${ }^{2}$, George Harauz ${ }^{1}$

Department of Molecular Biobgy and Genetics, and Biophysics Interdisciplinary Group, University of Gueph, "Molecular and Structural Biobgy, Ontano Cancer Institute, and Department of Medical Biaphysics, University of Toronto,

The ribosome is the protein synthetic machinery in the cell. Knowledge of the structures of ribosomal RNA (rRNA) macromolecules in situ is essential to understanding their roles in ribosome mediated protein synthesis. We are using a microanalytical technique that identifies and maps elements directly, electron spectroscopic imaging ${ }_{1}^{1,14}$ to determine the rRNA phosphorus distributions within Escherichia coli ribosomal subunits, and to combine the two-dimensional maps into a three-dimensional elemental distribution by iterative quaternion-assisted angular reconstitution ${ }^{11}$ of ribosomal particles at random orientations.

Elemental phosphorus maps represent the path of the nucleic acid backbone within nucleoprotein complexes. ${ }^{1-6,13,14}$ Nucleic acids (DNA, RNA) have phosphate backbones, while proteins generally have much less phosphorus. In E. coli, a prokaryote, ribosomal proteins are not phosphorylated at all. Localisation of rRNA in both the large and the small ribosomal subunits of this organism was demonstrated to be feasible over a decade ago by either electron spectroscopic imaging ${ }^{7,13}$ or scanning transmission electron microscopy. ${ }^{8,9}$ Our approach to electron spectroscopic imaging ${ }^{1,14}$ has a very high sensitivity, being capable of easily detecting the 300 phosphorus atoms in a single nucleosome, with a lower detection limit of about 30 phosphorus atoms. ${ }^{2,45}$ Briefly, images are formed using electrons with a narrow range of energy losses caused by specific inner shell ionisation interactions within the specimen. An image formed by $150 \pm 8 \mathrm{eV}$ energy loss electrons, in addition to the mass distribution, is augmented by a phosphorus signal because it also contains the $L_{2,3}$ ionisation peak of this

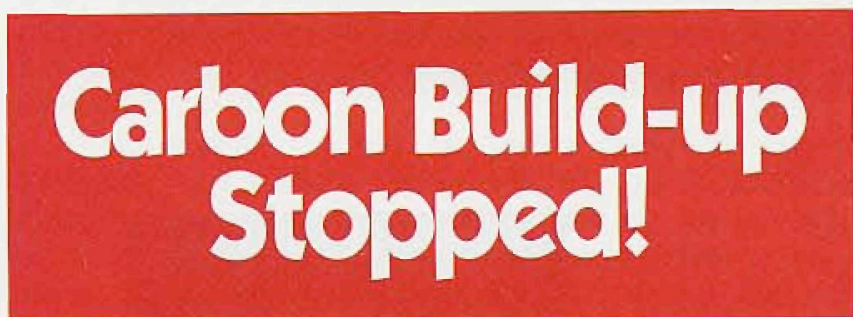

\section{SEM Laboratory Secret Revealed:}
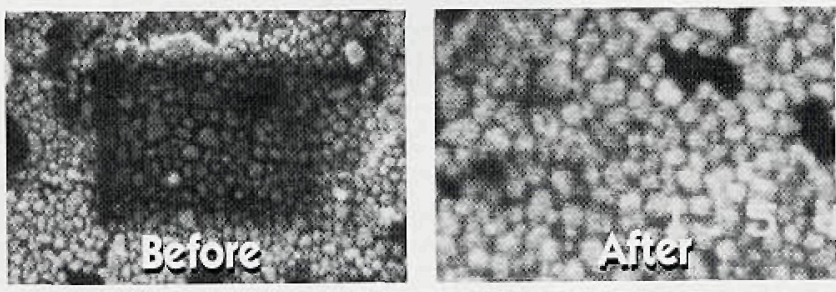

SEM manufacturers won't admit it, but most SEMs are subject to contamination build-up-even dry pumped systems. To stop hydrocarbon condensation, smart SEM users rely on the XEI Scientific SEM-CLEAN ${ }^{\text {TM }}$ system.

Result: Outstanding pictures at low $\mathrm{kV}$ and high resolution and no oil on EDS X-ray detector windows. The Nitrogen purge of the inexpensive SEM-CLEAN system cleans your electron microscope while you're away.

SEM-CLEAN"' Stops the Oil

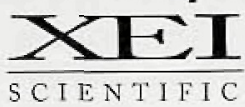

3124 Wessex Way, Redwood City, CA 94061

415-369-0133 * Fax 415-363-1659 element. These $150 \pm 8 \mathrm{eV}$ loss images thus present nucleic acid containing regions with enhanced contrast without the need for exogenous heavy metal stains or shadowing. A background image using $100 \pm 8 \mathrm{eV}$ loss electrons represents a non-specific mass distribution (of both protein and nucleic acid) of the sample. Computational subtraction of the $100 \mathrm{eV}$ loss image from the 150 $\mathrm{eV}$ loss image, after correction for exposure differences and film fog, can be used to form the net phosphorus (NetP) map of the specimen.

A recent advance which permits such images to be used to determine the three-dimensional structure of ribonucleoprotein complexes is based on the principle of common axis mediated angular reconstitution. ${ }^{11}$ The quaternionassisted angular reconstitution implementation that we utilise has been applied c recently to structure determination of numerous proteins and nucleoproteins. ${ }^{4,10} \frac{\bar{v}}{\bar{n}}$ In $150 \pm 8 \mathrm{eV}$ loss images of quick-frozen and freeze-dried $E$. coli ribosomal subunits, individual complexes within these images lie at essentially random $\stackrel{\circ}{\supset}$ orientations with respect to each other. Quaternion-assisted angular reconstitution is used to determine the relative angular orientations amongst different particles, enabling subsequent three-dimensional reconstruction. This method is $\frac{3}{3}$ robust in that it works well with relatively noisy individual images, allowing the use of smaller data sets. Three-dimensional reconstructions are then performed ${ }^{\text {D }}$ using a conventional algorithm of filtered back-projection. The three-dimensional reconstructions are then displayed and rotated interactively using INSIGHT II (BioSym Inc., Parsippany, NJ) molecular graphics software.

In the Cover Figure, the $150 \pm 8 \mathrm{eV}$ loss reconstructions of both subunits are merged computationally to give a representation of the whole ribosome with the large and small subunits positioned as they would be during translation. There is a large central cavity which provides sufficient room to contain 2 or 3 molecules of transfer RNA (tRNA) and the necessary ancillary protein factors. The cavity is also accessible to the exterior for admission and egress of these small macromolecules. The messenger RNA (mRNA) can pass through the hole in the neck of the NetP reconstruction of the small subunit, in full agreement with the interpretations of cryoelectron microscopical reconstructions. $^{12}$ The hole in the back of the reconstuctions of the large subunit can potentially be the outlet for the nascent polypeptide.

In spite of the higher irradiation demands of electron spectroscopic imaging not only for localizing, but also for identifying the phosphorus signal, the overall structures of the subunits in mass density and phosphorus reconstructions are consistent with features of cryoelectron microscopical reconstructions of the whole ribosome. ${ }^{12}$ Although phosphorus mapping of $E$. coli ribosomal subunits has been repeatedly demonstrated for over a decade, the present three-dimensional rRNA distributions in conjunction with the overall reconstructions are the first to be achieved for this nucleoprotein complex. This work demonstrates the potential of the combination of microanalytical electron spectroscopic imaging with computerized reconstruction for the study of the threedimensional structures of other nucleoprotein complexes.

\section{References}

Bauer R (1988) Meth. Microbiol. 20, 113-146.

2 Bazett-Jones DP (1993) Microbeam Anal. J. 2, 69-79.

3 Bazett-Jones DP, Leblanc B, Herfort M, Moss T (1994) Science 264, 1134-1137.

4 Bazett-Jones DP, Mendez E, Czarnota GJ, Ottensmeyer FP, Allfrey VG (1996) Nucl. Acids Res. 24, 321-329.

5 Bazett-Jones DP, Ottensmeyer FP (1981) Science 211, 169-170

6 Beniac DR, Harauz G (1995) Mol. Cell. Biochem. 148, 165-181

Boublik M, Oostergetel GT, Frankland B, Ottensmeyer FP (1984) In Bailey GW (ed), Proceedings of the 42nd Annual Meeting of the Electron Microscopy Society of America. San Francisco Press, San Francisco, pp 690-691.

8 Boublik M, Mandiyan V, Tumminia S, Hainfeld JF, Wall JS (1990) In Proceedings of the XIIth International Congress for Electron Microscopy. San Francisco Press, San Francisco, pp 134-135.

9 Boublik M, Wall J (1992) In Bailey GW, Bentley J, Small JA (eds.). Proceedings of the 50th Annual Meeting of the Electron Microscopy Society of America. San Francisco Press, San Francisco, pp 462-463.

10 Czarnota GJ Ottensmeyer FP (1996) J. Biol. Chem. 271, 3677-3683.

11 Farrow NA, Ottensmeyer FP (1993) Ultramicroscopy 52, 141-156, 1993.

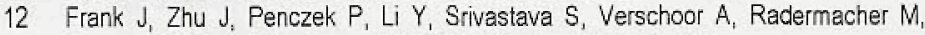
Grassucci R, Lata RK, Agrawal RK (1995) Nature 376, 441-444.

13 Korn AP, Spitnik-Elson P, Elson D, Ottensmeyer FP (1983) Eur. J. Cell Biol. 31. 334340

14 Ottensmeyer FP (1982) Science 215, 461-466 

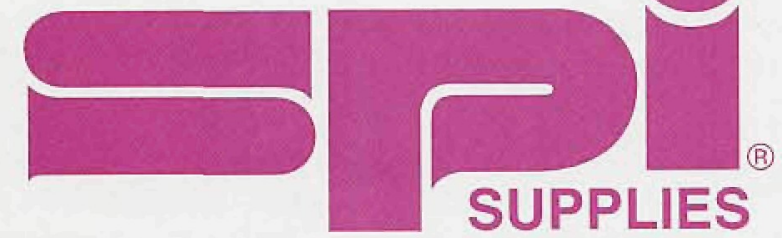

$\approx$ File Edit Diew Go Bookmarks 0ptions Directory Help

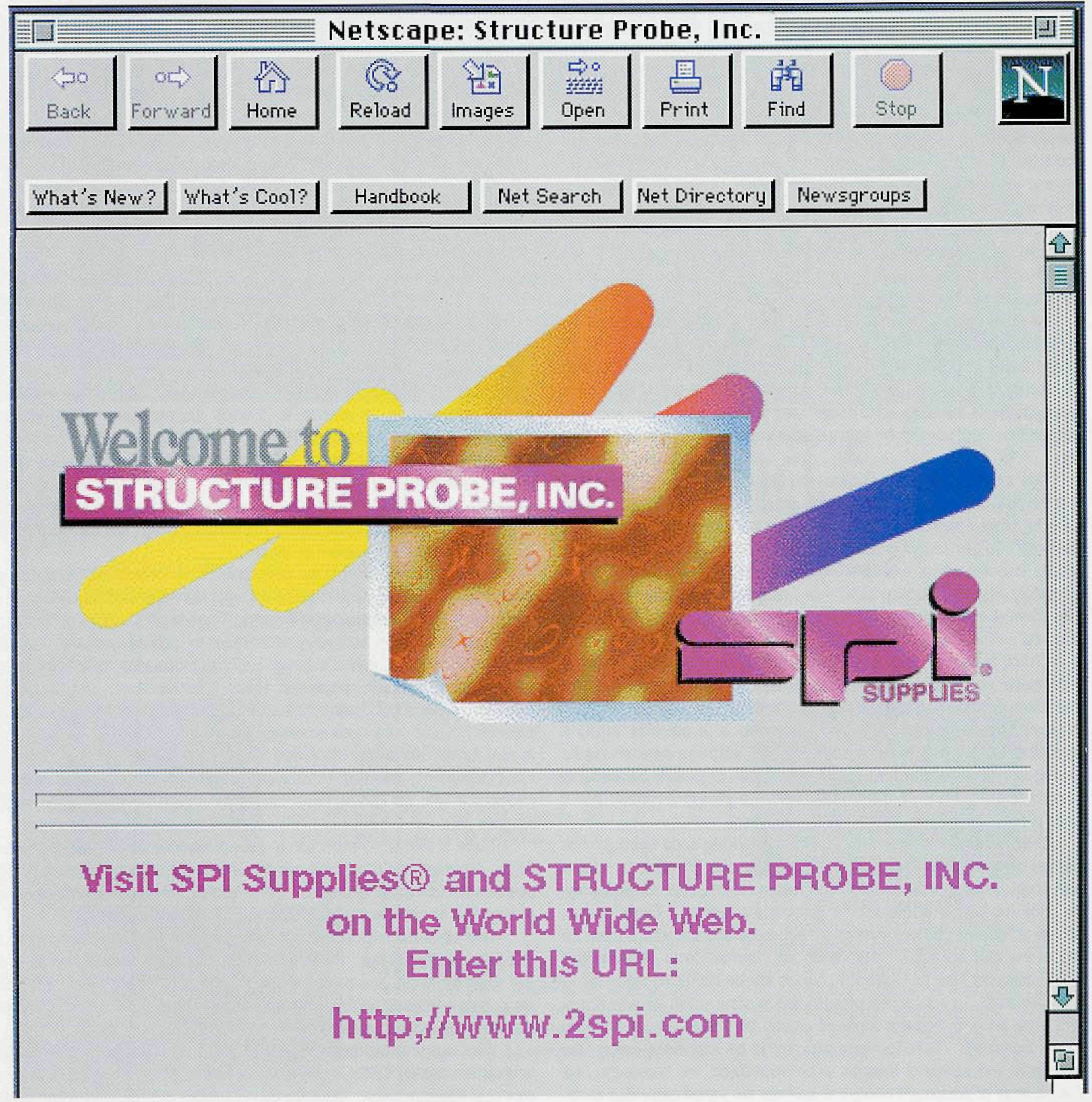

Look for our 1996 Sourcebook on the World Wide Web http://www.2spi.com

SPI Supplies Division of STRUCTURE PROBE, INC.

P.O. Box 656 - West Chester, PA 19381-0656 USA

Phone: 1-800-2424-SPI 1-610-436-5400 - FAX: 1-610-436-5755

e-mail: SpiSupp@aol.com •WWW at http://www.2spi.com 